\title{
Controllable Electromagnetic Signal Shielding Card Covers
}

\author{
Yun Sha \\ School of North China Electric Power University, Beijing 102200, China \\ y.sha@ncepu.edu.cn
}

Keywords: Near Field Communication, electromagnetic shielding, HFSS, S-parameter.

\begin{abstract}
NFC (Near Field Communication) technology is convenient for people's lives, but because of the abundant personal information contained in NFC cards, which can create a hidden danger for the leak of personal information. The existing NFC signal shielding cover is completely wrapped with a thin aluminum card, but this kind of wrapping will cause inconvenience to users. On the one hand, it covers cards and make cards invisible. On the other hand, when using, users need to remove the covers to let cards work normally. By HFSS simulation, this paper designed a new electromagnetic signal shielding transparent card cover, through changes of structural on copper ring to alter the strength of eddy current effect, realized the function of electromagnetic signal shielding controllable, and perfectly solved the existing technical problems.
\end{abstract}

\section{Introduction}

NFC (Near Field Communication) technology has become increasingly complete, and its related NFC card and read equipment also gradually come into all aspects of our life. NFC is convenient for people's lives, but because of cards containing abundant information, inconvenience and hidden trouble still exist when using.

First, the NFC card contains abundant information, buried a hidden danger for the leak of personal information [1]. For example, bank card can be read nearly 10 times consumption records by any mobile phone with NFC reading equipment. The private information theft may cause serious consequences.

Second, the existing NFC signal shielding card covers, using thin aluminum completely wrapped, shielding the read signal of electromagnetic field by utilizing the eddy current effect. But this kind of package way will cause inconvenience to users. On the one hand, it covers cards. When using, users have to remove the covers to recognize. On the other hand, due to the shielding of this cover is uncontrol, uses cannot use cards without removing covers.

Aiming at these problems, to realize visibility and controllability of the electromagnetic shielding card covers, author studied the working principle and characteristics of antenna of NFC, antenna transmission and matching design, electromagnetic wave transmission and field shielding, computing electromagnetic and so on. By using SMITH and HFSS, the author made simulations and tests on transmission of the electromagnetic signal resulted by NFC around the card, and chose eddy-current screen as the final way of shielding [2]. Considering the demands of shielding performance, visibility, the author chose the high conductivity material, copper, designed different visibility of shielding structure, testing various shielding structure one by one, and finally determined the structure of copper ring to realize shielding. Apply the principle of eddy-current effect to make card covers controllable. When copper ring is closed, it provides a complete current channel for the current produced by radio frequency signal, which can play a good role in shielding. When copper ring is disconnected, the current circuit is broken, which greatly reduces the induced current. And then, shielding card covers will not affect the normal use of NFC cards. 


\section{Methods}

\subsection{Electromagnetic Structure Model.}

Based on the characteristics of antenna, abstract the working principle of NFC as an electromagnetic structure model showed in Fig. 1 [3].
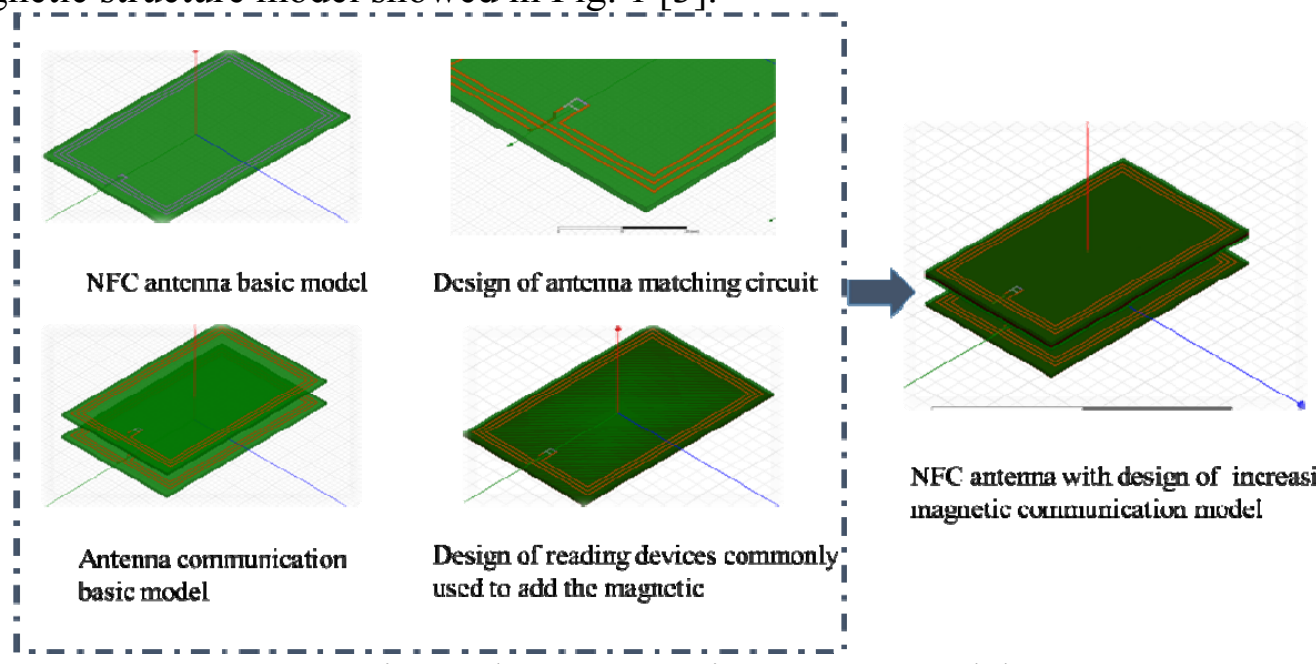

Fig. 1 Electromagnetic structure model

Through the design of NFC antenna matching circuit, combination with commonly used magnetic structure, eventually choose series matching circuit and determine the antenna design parameters which are shown in Table 1.

Table 1. The antenna design parameters

\begin{tabular}{cc}
\hline Parameter Name & Value of Parameter \\
\hline long side of coil's outside edge & $80 \mathrm{~mm}$ \\
short side of coil's outside edge & $50 \mathrm{~mm}$ \\
width of the coil & $0.5 \mathrm{~mm}$ \\
turns of the coil & 3 \\
space between two turns & $1 \mathrm{~mm}$ \\
series matching resistance & $12,5 \Omega$ \\
series matching capacitance & $32.0 \mathrm{pF}$ \\
parallel matching capacitance & $32.7 \mathrm{pF}$ \\
thickness of magnetic piece & $0.1 \mathrm{~mm}$ \\
\hline
\end{tabular}

To make the design of the NFC antenna can effectively reflect the actual antenna operating characteristics, select the inductance of antenna, the resonance frequency of electromagnetic performance to do tests. The results show that the antenna can work on $13.56 \mathrm{MHz}$ [3].

\subsection{Shielding Principle.}

After discussing and researching shielding ways in the table below, the author chose eddy-current screen as the final shielding way [4].

Table 2. Shielding ways

\begin{tabular}{|c|c|c|c|c|}
\hline $\begin{array}{l}\text { Electromagnetic } \\
\text { Shielding Type }\end{array}$ & Electronic Shielding & Magneti & c Shielding & Electromagnetic Shielding \\
\hline subtype & electrostatic & electrostatic & alternating & electromagnetic shielding \\
\hline $\begin{array}{c}\text { key point } \\
\text { usable range }\end{array}$ & $\begin{array}{l}\text { conductor cavity coupling capacity } \\
\text { electrostatic field alternating field }\end{array}$ & $\begin{array}{l}\text { high permeability } \\
\qquad<100 \mathrm{kHz}\end{array}$ & $\begin{array}{l}\text { eddy-current effect } \\
\quad>100 \mathrm{MHz}\end{array}$ & $\begin{array}{l}\text { assimilation and reflection of shield } \\
\text { dynamic electromagnetic field }\end{array}$ \\
\hline
\end{tabular}

\subsection{The Selection of Shielding Indicator.}

$\mathrm{S}$ parameter, a scattering parameter, is an important parameter in microwave transmission. $S_{i j}$ Represent the meaning that energy measured in $i$ port when it is injected from $j$ port. In the two-port network, $S_{12}$ is called reverse transmission coefficient, namely isolation. $S_{21}$ is called positive transmission coefficient, namely gain. $S_{11}$ is called input reflection coefficient, namely input return loss, $S_{22}$ is called output reflection coefficient, namely output return loss. For reciprocal network, $S_{12}=S_{21}[5]$. 
For the air and shielding structure in the middle of the NFC card and read equipment, they can be equivalent to a two-port network, reading termination input signal, card termination output signal. If let Port1 as the signal input port, Port2 as the signal output port, then $S_{11}$ represents the return loss, namely how much energy can be reflected to the source side (Port1), the value of which the smaller the better. $S_{21}$ Represents insertion loss, that is, how much energy is transferred to the destination side (Port2), when the transmission energy is less than a certain value, the device will not be able to work properly. Through the comparison of the simulation calculation and experiment in the NFC card reading situation, when S parameters is bigger than $15 \mathrm{Db}$, NFC cards can be normal use, when $S_{21}$ is smaller than $20 \mathrm{~dB}$, reading equipment is unable to read information from the NFC card properly.

\section{Simulation and Conclusion}

\subsection{Selection of Shielding Materials.}

After taking the theory of eddy-current screen, the selection of high conductivity materials for shielding should be essential. Obviously, these materials, due to the characteristic of invisible, affect the normal use of cards. To this end, the author chose to adopt high electric conductivity materials to ensure the shielding effectiveness after reducing the size of shield.

Table 3 S-parameters of different shielding materials

\begin{tabular}{cc}
\hline Shielding Material & S-parameter \\
\hline unshielded & $-8.27 \mathrm{~dB}$ \\
$\mathrm{Al}$ & $-39.7 \mathrm{~dB}$ \\
$\mathrm{Fe}$ & $-33.2 \mathrm{~dB}$ \\
$\mathrm{Cu}$ & $-48.5 \mathrm{~dB}$ \\
$\mathrm{Si}$ & $-8.5 \mathrm{~dB}$ \\
solid mask & $-8.55 \mathrm{~dB}$ \\
\hline
\end{tabular}

From the data shown in Table 3, it is easy to find copper and aluminum have higher electric conductivity.

\subsection{Optimization of Shielding Structure-Considering Visibility.}

Because of the inconvenient using experiment caused by traditional invisible card covers, make covers be visible could facilitate the lives of people.

After choosing copper, aluminum in the first place, put forward the following shielding structure by considering making the shield meet the requirement of visibility by reducing the size of card cover material.

Table 4. Shielding structure and parameters

\begin{tabular}{ccc}
\hline Form of Shielding & Visible Area & S-parameter \\
\hline $\mathrm{Cu}$ - plate like & $0 \%$ & $-48.5 \mathrm{~dB}$ \\
$\mathrm{Al}$ - plate like & $0 \%$ & $-39.7 \mathrm{~dB}$ \\
$\mathrm{Cu}$ - hollowed-out & $50 \%$ & $-43.4 \mathrm{~dB}$ \\
$\mathrm{Cu}$ - half plate covering & $50 \%$ & $-10 \mathrm{~dB}$ \\
$\mathrm{Al}$ - half plate covering & $50 \%$ & $-8.6 \mathrm{~dB}$ \\
$\mathrm{Cu}$-netlike & $70 \%$ & $-42.2 \mathrm{~dB}$ \\
$\mathrm{Cu}$-ring like & $80 \%$ & $-30.10 \mathrm{~dB}$ \\
\hline
\end{tabular}

From the simulation results showed in Table (), the conclusion which we can draw is that when reducing the shielding area, shielding ability will fall sharply. When the area is less than $50 \%$, the cover material is unable to meet the requirement of shielding. When using the netlike, hollowed-out and ring covering shielding structure, the shielding ability will not fall a lot. So, a preliminary judgment is that the netlike and ring like covering shielding structure can both meet the requirements of shielding ability and visibility.

\subsection{Optimization of Shielding Structure-Considering Controllability.}

When using traditional shielding card covers. Users have to remove the covers to ensure NFC cars working normally. So, if the covers are controllable, it will make cards convenient to use. 
Contact the requirement of visibility and consider controllability, some shielding schemes for choice are listed in Table 5.

Table 5. Shielding scheme

\begin{tabular}{cccc}
\hline Form of Shielding & Visible Area & S-parameter (before acting) & S-parameter (after acting) \\
\hline unmask & $100 \%$ & $-8.27 \mathrm{~dB}$ & - \\
$\mathrm{Cu}$ - plate like & $0 \%$ & $-48.5 \mathrm{~dB}$ & - \\
$\mathrm{Cu}$ - half plate covering & $50 \%$ & $-10 \mathrm{~dB}$ & $-12.2 \mathrm{~dB}$ \\
3mm Cu ring like & $92 \%$ & $-26.5 \mathrm{~dB}$ & $-8.57 \mathrm{~dB}$ \\
$5 \mathrm{~mm}$ Cu ring like & $71 \%$ & $-37.0 \mathrm{~dB}$ & $-9.22 \mathrm{~dB}$ \\
\hline
\end{tabular}

From the simulation and experiment results, author found that the shielding effectiveness of eddy-current screen depends on the size of the area surrounded by circulation. The statistics indicate that the copper ring like form of shielding has the multiple advantages of visibility, controllability and economy.

\subsection{Conclusion.}

Through the above simulation for many times, more detailed results are listed here. First, the shielding material and the structure should adopt copper and ring like form. Second, the suitable width of the copper ring is $3 \mathrm{~mm}$. Third, considering insulation, only use one turn of copper ring.

Use copper ring to shield can satisfy the requirements of visibility, controllability, and has the advantages of less material consumption, occupied less space, simple producing process, low cost, etc.



Fig. 2 Copper ring is closed

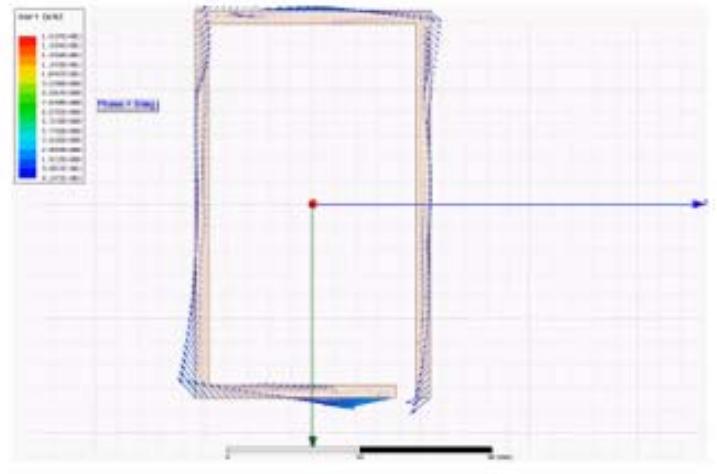

Fig. 3 Copper ring is disconnected

The simulation results show that the closed ring of copper provides a complete current channel for current produced by radio-frequency signal, which has played a good role in shielding. When shield ring is disconnected, the current circuit is broken, which greatly reduces the induced current. And then, shielding card covers will not affect the normal use of NFC cards.

\section{References}

[1]. Jun Yang. NFC technology and its application, standard progress and test[J]. Modern Telecommunications Technology. 2009, 39(10):1-5.

[2]. Mingyang Li. HFSS electromagnetic simulation and design application [M]. People's posts and telecommunications press, 2010.

[3]. Yuanna Wei: Antenna design method based on the NFC technology (Master, Nanjing Post and Communications University, China 2012). p.5.

[4]. Hromádka I M. shielding effectiveness [J]. 2000:1569-1569.

[5]. Eisenstadt W R, Eo Y. S-parameter-based IC interconnect transmission line characterization [J]. Components Hybrids \& Manufacturing Technology IEEE Transactions on, 1992, 15(4):483-490. 